\title{
人外周血单核细胞来源的树突状细胞的体外 “2+2”快速法培养及其鉴定
}

\author{
陆小鹏 ${ }^{1,3}$, 李 剑 ${ }^{2}$, 孙正华 ${ }^{1,3}$, 赵 娜 ${ }^{1,3}$, 刘 洁 $^{1}$, 张华堂 ${ }^{1, *}$ \\ (1. 中国科学院昆明动物研究所 免疫生物学实验室, 云南 昆明 650223 ; 2. 中国科学院昆明动物研究所 中心实验室,
}

云南 昆明 650223；3. 中国科学院研究生院, 北京 100049)

摘要: 以人浓缩白细胞来源的 $\mathrm{CD} 14^{+}$单核细胞为前体, 建立体外快速培养树突状细胞 (dendritic cell, DC) 的 方法。采用密度梯度离心和 MACS 磁珠分选系统, 收集高纯度的 CD14 ${ }^{+}$单核细胞; 以 rGM-CSF、rIL-4 联合分化 2 天诱导不成熟 DC, 再将分化后的细胞以 rTNF- $\alpha$ 、IL-1 $\beta 、 I L-6 、 \mathrm{PGE}_{2}$ 共同活化 2 天得到成熟 DC。流式细胞仪 检测结果表明, 分化 2 天的不成熟 DC 具有吞噬能力, 且表型 HLA-DR、CD40、CD80 表达在 80\%以上, CD83、 CD86 基本不表达，成熟后的 DC 能够激活 $\mathrm{T}$ 细胞增殖，HLA-DR 表达增高，CD83、CD86 表达占 $85 \%$ 。

关键词: 单核细胞; 树突状细胞; “ $2+2$ ”快速法

中图分类号: Q25 文献标识码: A 文章编号：0254-5853-(2008)04-0415-06

\section{A “ $2+2$ days” Fast Protocol for the Generation of Dendritic Cells from Human Blood Monocytes}

\author{
LU Xiao-peng ${ }^{1,3}$, LI Jian ${ }^{2}$, SUN Zheng-hua ${ }^{1,3}$, ZHAO Na ${ }^{1,3}$, LIU Jie ${ }^{1}$, ZHANG Hua-tang ${ }^{1, *}$ \\ (1. Immunobiology Lab, Kunming Institute of Zoology, the Chinese Academy of Science, Kunming 650223, China; 2. Core Faculty, Kunming Institute of
}

Zoology, the Chinese Academy of Science, Kunming 650223, China; 3. Graduate School of the Chinese Academy of Science, Beijing 100049, China)

\begin{abstract}
A " $2+2$ days" fast protocol for the generation of dendritic cells( DC) from high purity human monocytes in vitro has been established. During the 2-step differentiation and activation process, we demonstrated that 2 days of culture with GM-CSF and IL-4 were sufficient to generate immature DCs capable of antigen uptake. Similarly the mature DCs were activated with a cocktail of rTNF- $\alpha$, IL-1 $\beta$, IL-6, and PGE $_{2}$ from immature DC in 2 days. This " $2+2$ " fast DC had the same phenotype and function as the " $6+2$ " standard DC.
\end{abstract}

Key words: Monocyte; Dendritic cell; “ $2+2$ days” fast method

树突状细胞（dendritic cell, DC）是体内高效 的抗原递呈细胞（antigen presenting cel1, APC）。 它们以未成熟状态分布于机体的皮下或黏膜组织 内，通过识别捕获微环境内的“异己”成分，将其加 工并携带至淋巴组织, 完成抗原呈递和 DC 成熟的 过程, 进而激活静息 $\mathrm{T}$ 淋巴细胞（resting $\mathrm{T}$ cell）应 答, 启动免疫反应（Sousa，2006）。

目前, 在基础研究和临床应用领域, DC的获得 主要有 3 种方式: (1)直接从外周血分离; (2)从脐带 血和骨髓来源的 $\mathrm{CD} 34^{+}$细胞诱导; (3)由外周血来源
的CD14 ${ }^{+}$单核细胞 (monocyte) 诱导 (Tuyaerts et al, 2007）。由于外周血DC含量不到 $1 \%$, 脐带血和骨 髓中 CD $34^{+}$细胞仅有 $1 \%$ 左右 (Jeras Bergant \& Repnik，2005），而外周血中CD14 单核细胞达 15 \%左右 (Campbell-AnsonKentor \& Radvanyi 2008), 所以通常选择 $\mathrm{CD} 14^{+}$单核细胞为前体，按“ $6+2$ ”传 统法, 经过分化和活化两个阶段诱导DC (standard $\mathrm{DC}$ ）。即将贴壁收集的 $\mathrm{CD} 14^{+}$单核细胞，用分化因 子诱导 5-7天得到具有抗原摄取能力的不成熟 $\mathrm{DC}$ (immature DC, iDC), 再用活化因子刺激 $2-3$ 天得

收稿日期: 2008-04-03; 接受日期: 2008-06-17

基金项目: 国家自然科学基金面上项目 (30771951); 中国科学院 “百人计划”项目; 中国科学院知识创新工程重要方向项目 (KSCX1-YW-R-16) “通讯作者 (Corresponding author), E-mail: zhanght@post.kiz.ac 第一作者简介: 硕士研究生, E-mail: luxiaopengcas@yahoo.cn 
到能够激活 $\mathrm{T}$ 细胞增殖的成熟 DC (mature DC, mDC) (Romani et al,1996; Zhou \& Tedder, 1996)。

由于 $\mathrm{CD} 14^{+}$单核细胞向 $\mathrm{DC}$ 分化的过程中并不 发生增殖, 随着体外培养时间的延长细胞大量死 亡, 传统法诱导 $\mathrm{DC}$ 的得率相对较低。另一方面, $\mathrm{DC}$ 为终末分化细胞, 无法在体外形成稳定的细胞 系。传统法极大的制约了 $\mathrm{DC}$ 的实验研究, 其在 DC 诱导周期与得率两方面的不足亟待改进。

而在体内, $\mathrm{DC}$ 前体细胞分化成DC只需要 $1-4$ 天, Randolph et al (1998)、Santini et al (2000) 和 Landi et al (2007) 等以此为基础, 在体外培养体系 中加入内皮细胞、I类干扰素, 缩短了体外诱导 DC 的时间周期。Dauer在前人研究的基础上, 提出了 48h “Fast DC”快速诱导DC模型 (Dauer et al, 2003)。 但其成熟阶段的特征表型 $\mathrm{CD} 83$ 表达不高, 小于 60 \% (Obermaier et al, 2003)。其可行性还存有较大争 议, 所以在 $\mathrm{DC}$ 的体外培养过程中, 诱导所需的确切 时间以及培养条件仍需进一步探讨。

为此, 本研究利用 MACS 磁珠分选系统, 从人 浓缩白细胞中分选出高纯度的 $\mathrm{CD} 14^{+}$单核细胞, 分 阶段用 rGM-CSF、rIL-4 分化, rTNF- $\alpha$ 、IL-1 $\beta$ 、IL-6、 $\mathrm{PGE}_{2}$ 联合活化, 定向诱导出在表型和功能上与传统 法相一致的 DC。建立了一种快捷、可靠的体外“2 +2 ”培养 DC（fast DC）的优化方法。

\section{1 材料方法}

\section{1 试 剂}

RPMI1640 培养基, 胎牛血清为 GIBICO 公司 产品; 淋巴细胞分离液为上海恒信有限公司产品; CD14 MicroBeads human monocyte kit 德国 Miltenyibiotec 产品; rGM-CSF、rIL-4、rTNF- $\alpha$ 购 自 R\&D 公司; IL-1 $\beta$ 、IL-6 为 Peprotech 公司产品; $\mathrm{PGE}_{2}$ 为 Alexis 公司产品。 $\mathrm{PE}$ 标记的 CD80、CD86、 CD83、HLA-DR、CD14、CD40、CD1a 及同型对 照 IgG1、IgG2b、IgG2a 购于 BioLegend 公司; Monoclonal Anti-human DC-SIGN-Phycoerythrin 购 于 R\&D 公司; FITC-dextran 和 CFSE 染料购自 Sigma 公司。

\section{2 仪器设备}

LSM510 META激光共聚焦扫描显微镜（德国 Zeiss 公司), FACS Vantage SE流式细胞分析仪 (BD 公司), HEPA Class100细胞培养箱和Forma ClassII A2生物安全柜（Thermo公司）, Nikon TS 100倒置
相差显微镜（Nikon公司）。

\section{$1.3 \mathrm{CD}^{+} 4^{+}$单核细胞收集}

采用淋巴细胞分离液密度梯度离心法, 从浓缩 白细胞（取自昆明市血液中心）收集外周血单个核 细胞 ( PBMC); 按 CD14 MicroBeads human monocyte kit 操作说明, 分离收集 $\mathrm{CD} 14^{+}$单核细胞, 并将 $\mathrm{CD} 14^{-}$细胞冻存用于 $\mathrm{T}$ 细胞增殖实验。

\subsection{DC 诱导}

将 $\mathrm{CD} 14^{+}$单核细胞按 $1 \times 10^{6} \mathrm{cells} / \mathrm{mL}$ 接种于 6 孔 板中, 用 $3 \mathrm{~mL}$ 含 $10 \%$ 胎牛血清、 $2 \mathrm{mmol} / \mathrm{L}$-谷氨酰胺、 $100 \mathrm{U} / \mathrm{mL}$ 青霉素和 $100 \mu \mathrm{g} / \mathrm{mL}$ 链霉素的RPMI1640完 全培养基培养，并加诱导因子 rGM-CSF（1000 $\mathrm{U} / \mathrm{mL}) 、 \mathrm{rIL}-4(500 \mathrm{U} / \mathrm{mL}), 5 \% \mathrm{CO}_{2}, 37^{\circ} \mathrm{C}$ 条件下 培养。每隔2天换半液, 保持细胞因子终浓度一致, 分别诱导 2 天或 6 天后加入成熟诱导因子 rTNF- $\alpha$ (10 $\mathrm{ng} / \mathrm{mL}) 、 \mathrm{IL}-1 \beta(10 \mathrm{ng} / \mathrm{mL}) 、 \mathrm{IL}-6(10 \mathrm{ng} / \mathrm{mL}) 、 \mathrm{PGE}_{2}$ $(1 \mu \mathrm{g} / \mathrm{mL})$ 培养2天。

\section{5 流式检测细胞表型}

采用 $1000 \mathrm{r} / \mathrm{min}$ 离心 $10 \mathrm{~min}$, 分别收集上述培 养时间的细胞，再用含 $0.1 \% \mathrm{BSA}$ 的 PBS 冲洗封闭 细胞一次, 离心去上清后将细胞按 $5 \times 10^{5} / 50 \mu \mathrm{L}$ 重 悬于 $1.5 \mathrm{~mL}$ 的 $\mathrm{EP}$ 管中, 加入 $2 \mu \mathrm{L}$ 苂光标记的抗体, 常温下染色 $20 \mathrm{~min}$, 离心并取 $300 \mu$ LPBS 把染色后 的细胞转移于流式管, 用于流式细胞仪检测, 软件 WinMDI 2.9 分析数据。

\subsection{FITC-dextran 摄取检测}

收集单核细胞, 不成熟 DC 和成熟 DC 分别按 $1 \times 10^{6} \mathrm{cells} / \mathrm{mL}$ 悬于 RPMI1640 完全培养基中, 再加 入 FITC-dextran $(0.5 \mathrm{mg} / \mathrm{mL}), 37^{\circ} \mathrm{C}$ 培养箱或 $0^{\circ} \mathrm{C}$ 捊 育 $30 \mathrm{~min}, 1000 \mathrm{r} / \mathrm{min}$ 离心 $10 \mathrm{~min}$ 收集细胞, 用 $\mathrm{PBS}$ 清洗 3 次, 最后重悬于流式管, 分析检测细胞的苂 光强度, 以 $0^{\circ} \mathrm{C}$ 条件为基准, 判断细胞的抗原摄取 能力。

\section{7 混合淋巴细胞增殖反应}

将冻存的 $\mathrm{CD} 14^{-}$细胞复苏用于做同种异体 $\mathrm{T}$ 细 胞增殖实验。 $1000 \mathrm{r} / \mathrm{min}$ 离心 $10 \mathrm{~min}$, 收集复苏 1 天的 $\mathrm{CD} 14^{-}$细胞, $\mathrm{PBS}$ 洗两遍, $37^{\circ} \mathrm{C}$ 标记 CFSE (羟 基荧光素二醋酸盐琥珀酰亚胺脂）染料 $10 \mathrm{~min}$, $2 \times 10^{5}$ cells $/ 200 \mu \mathrm{L}$ 植于 96 孔板, 再按 $1: 1 、 1: 10$ 、 $1: 100 、 1: 1000\left(\mathrm{DC} / \mathrm{CD} 14^{-}\right)$加入成熟 $\mathrm{DC}$ ，共 同培养 3 天后, 收集细胞, 做流式检测, 以 CFSE 苂光强度的衰减次数显示细胞增殖变化。 


\section{2 结 果}

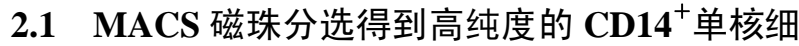 胞}

将经淋巴分离液离心收集的 PBMC 与 $\mathrm{MACS}$ 磁珠分选后的细胞分别标记 CD14 荧光抗体, 流式 检测表明在 PBMC 中 $\mathrm{CD} 14^{+}$单核细胞约占 $11 \%$ (图 1a)，而磁珠分选后 $\mathrm{CD} 14^{+}$单核细胞纯度达 $95 \%$ 以 上 (图 1c)。

\section{2 “2+2”快速法与“6+2”传统法诱导 DC 的形}

\section{态观察比较}

CD14 磁珠分选收集的单核细胞中加入 rGM-CSF、rIL-4 联合分化 2 天或 6 天 (iDC)，再 用成熟刺激因子活化 2 天 (mDC)。在传统法成熟 过程中, 选择加入 LPS 或 rTNF- $\alpha$ 或 rTNF- $\alpha$ 、IL- $1 \beta$ 、
IL-6、 $\mathrm{PGE}_{2}$ 联合活化，其中 LPS 或 rTNF- $\alpha$ 单独作 用时成熟 DC 的特征表面分子 CD83 表达不高（数 据未给出), 而 rTNF- $\alpha$ 、IL-1 $\beta$ 、IL-6、 PGE $_{2}$ 联合 作用后 $\mathrm{CD} 83$ 表达在 $85 \%$ 以上（图 3e ），所以在快 速法诱导的成熟过程中选择此 4 种因子共同活化。 从形态变化来看, $\mathrm{CD} 14^{+}$单核细胞呈圆形 (图 2a), 经 rGM-CSF 和 rIL-4 的诱导 2 天后, 细胞聚集成大 小不均的集落, 并在细胞表面有少许的突起（图 2b), 到 6 天后, 细胞贴壁, 外形不规则, 表面有 少许明显的细刺状突起 (图 2c ), 经 rTNF- $\alpha$ 、 IL- $1 \beta$ 、 IL-6、PGE 2 成熟刺激 2 天后, 细胞表现出典型的成 熟 DC 形态, 悬浮细胞增多, 体积变大, 表面长出 大量细刺状的触须 (图 2d、e、f)。“2 $2+2$ ”快速法诱 导的不成熟 DC 形态不及“6+2”传统法明显, 而成 熟 DC 形态基本一致。
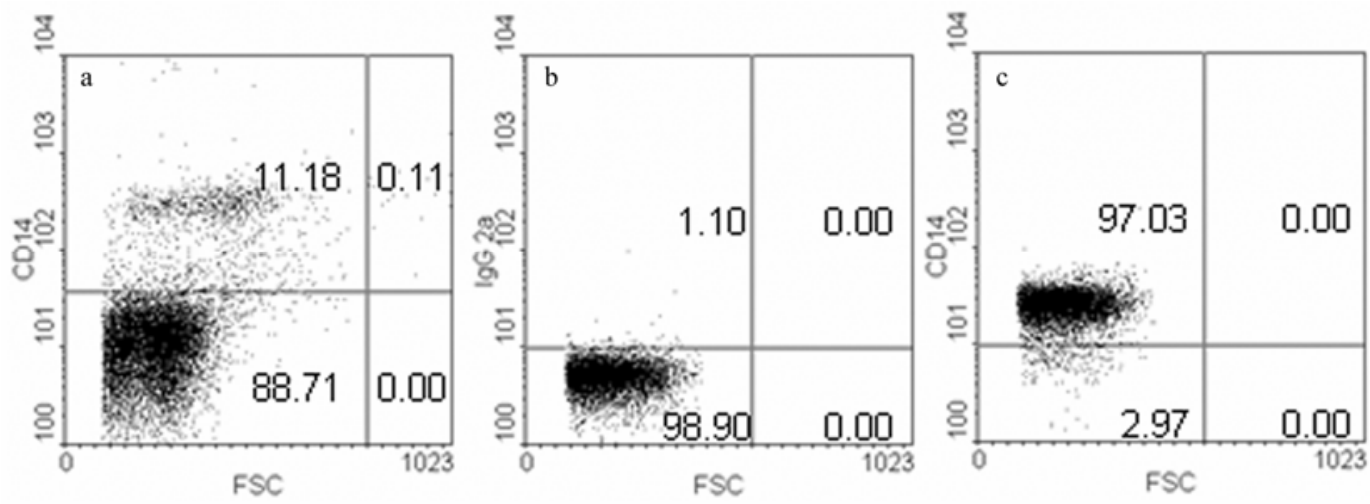

图 $1 \mathrm{CD} 14^{+}$单核细胞在磁珠分选前后的含量

Fig. 1 The purity of monocytes before and after MACS selection

a: PBMC; b: 分选后细胞同型对照 Isotype control; c: 分选后细胞纯量 Purity post selection.
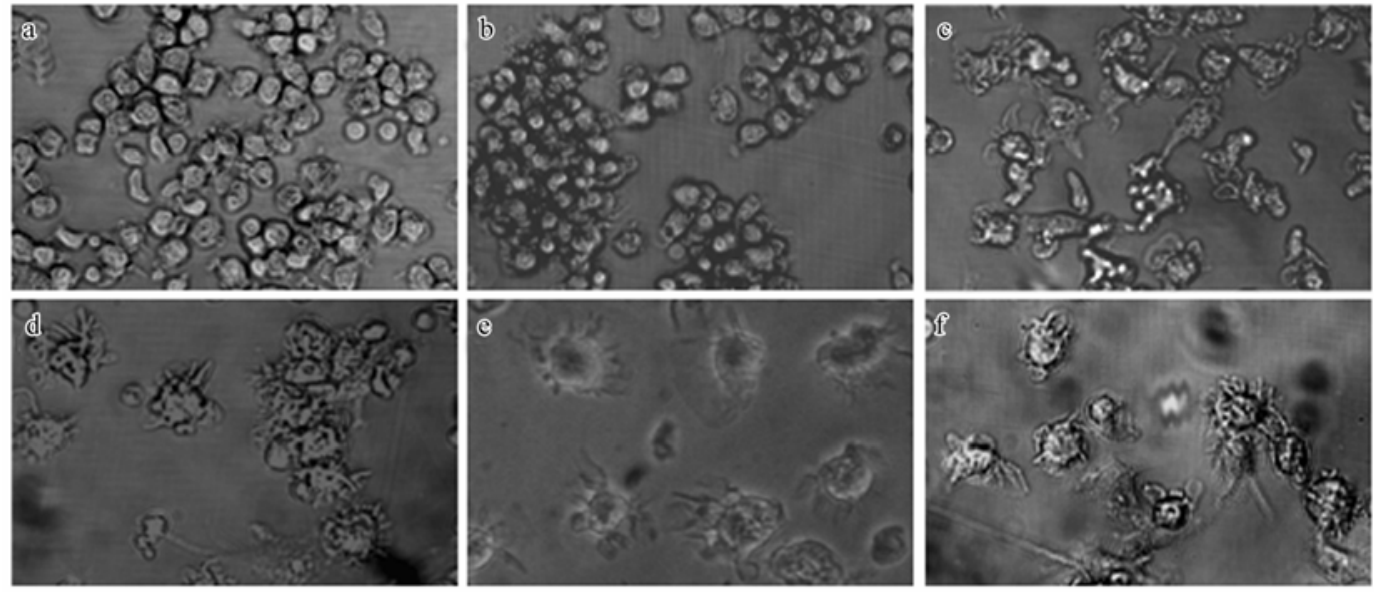

图 2 DC 在诱导分化过程中的形态变化

Fig. 2 The morphology of monocytes and DC

a: 单核细胞; b: 诱导 2 天不成熟 DC; c: 诱导 6 天不成熟 DC; d、e: 2+2 成熟 DC, f: $6+2$ 成熟 DC。

(a $-d$ 、f: 共聚焦扫描图片 $\times 200$; e: 倒置显微镜照片 $\times 200)$ 。

a: monocyte, b: 2d iDC, c: 6d iDC and d, e: fast mDC, f: standard mDC. (a-d, f: laser confocal scaning, $\times 200$; e: invert microscope, $\times 200$ )。 
2.3 单核细胞、不成熟 DC、成熟 DC 的表型分析 利用流式细胞仪检测单核细胞和DC细胞表面 分子的结果表明, 刚分离的 $\mathrm{CD} 14^{+}$单核细胞表达 HLA-DR、CD80，低表达DC-SIGN，不表达CD86、 CD83、CD40、CD1a（图3a）。经2天或6天刺激后 的iDC, CD14明显下调, HLA-DR、CD40、CD1a、 CD80、CD86、DC-SIGN 上调, CD83基本无变化 (图 $3 \mathrm{~b} 、 \mathrm{~d})$ 。经 2 天成熟诱导后的 $\mathrm{mDC}$, 基本不表达 CD14，高表达HLA-DR、CD40、CD80、CD86、 CD83、中度表达CD1a和DC-SIGN（图3c、e)。快 速法与经典法在表面标志上的差别在于, 在 $i D C$ 分 化阶段, “2 +2”法下调 CD14, 上调 HLA-DR、 DC-SIGN的程度不及传统法明显 (图3b、d); 而 $\mathrm{mDC}$ 活化阶段, DC-SIGN和CD1a的下调, 快速法更加明 显 (图3c、e); 但在诱导DC的全部过程中, 两种方
法影响表型变化的趋势一致, CD14表达明显减低, HLA-DR、CD86和CD83在成熟阶段表达显著增强, CD1a、DC-SIGN集中表达在不成熟阶段，而后则下 调; CD40在不成熟阶段的表达量与成熟阶段基本维 持不变。

\section{4 不成熟 $\mathrm{DC}$ 的吞噬功能}

抗原吞噬能力是 $\mathrm{iDC}$ 的主要功能之一, 为此用 流式的方法比较单核细胞、 $\mathrm{iDC}$ 和 $\mathrm{mDC}$ 在 $37^{\circ} \mathrm{C}$ 条件 下对Dextran的摄取能力的差异，结果证实，iDC具 有吞噬功能 (图 $4 \mathrm{~b} 、 \mathrm{~d}$ )，而 $\mathrm{mDC}$ 吞噬能力丧失（图 $3 c 、 e)$; 单核细胞有微弱的吞噬能力 (图3a)。两种 方法诱导的iDC吞噬能力基本相同，在成熟过程中 吞噬能力的变化也基本一致（图4b、c)。

\section{5 成熟 DC刺激 $\mathrm{T}$ 细胞增殖反应}

iDC在摄入抗原后, 逐渐成熟并迁移到T淋巴细

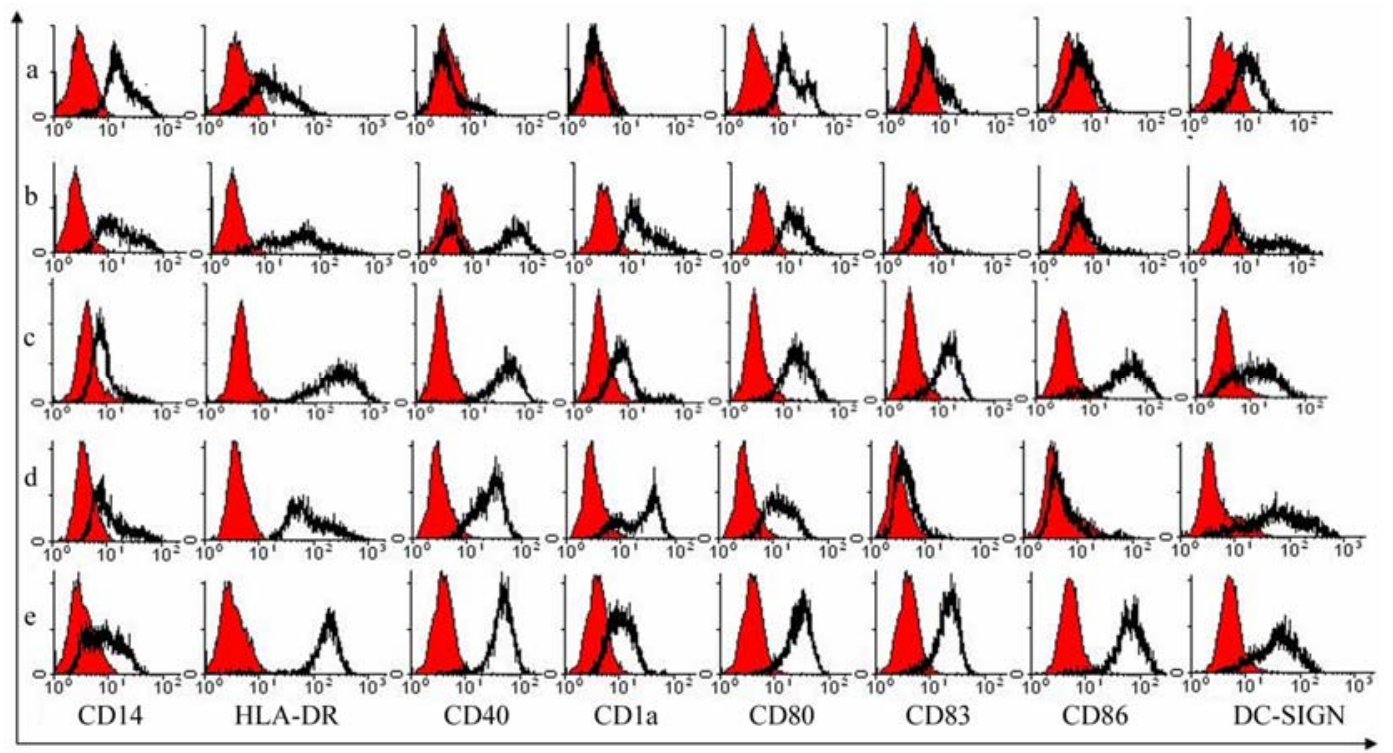

图 3 传统法与快速法诱导 DC 的表面标志流式检测变化

Fig. 3 The characteristic phenotype of fast DC versus stand DC

a: 单核细胞; b: 2 天不成熟 DC; c: $2+2$ 成熟 DC; d: 6 天不成熟 DC，e: $6+2$ 成熟 DC。

a: monocyte; b: fast iDC; c: fast mDC; d: standard iDC; e: standard mDC.

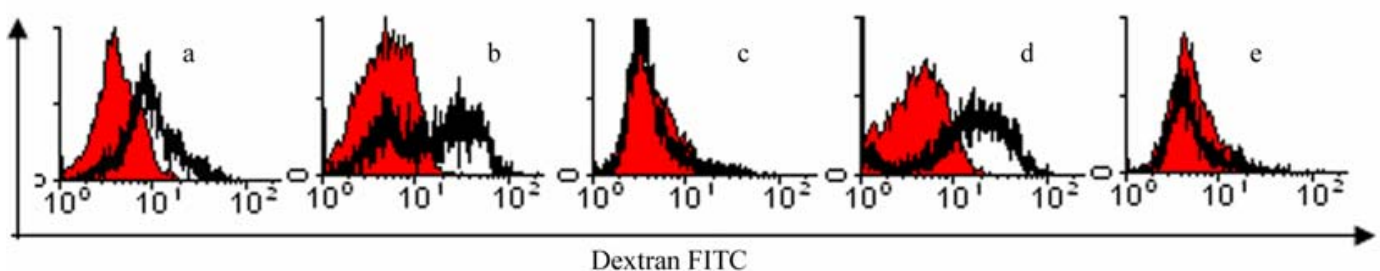

图 4 DC 对 Dextran 摄取能力变化

Fig. 4 Dextran uptake capacity of DC

$\mathrm{a}$ : 单核细胞、 $\mathrm{b}: 2$ 天不成熟 DC, c: $2+2$ 成熟 DC， d: 6 天不成熟 DC, e: 6+2 成熟 DC。 a: monocyte,,b: fast iDC, c: fast $\mathrm{mDC}, \mathrm{d}$ : standard iDC, e: standard $\mathrm{mDC}$. 
胞区域, 递呈抗原刺激 T细胞增殖引发免疫反应。 本实验也同时用混合淋巴细胞培养 ( Mix Lymphocyte Reaction, MLR）分析比较了两种方法 诱导的 $\mathrm{mDC}$ 激活 $\mathrm{T}$ 细胞增殖能力的差异。发现两种
方法诱导的 $\mathrm{mDC}$ 在激活 $\mathrm{T}$ 细胞的能力上基本相同, $\mathrm{DC}$ 与 $\mathrm{PBMC}$ 共同培养3天后, $\mathrm{PBMC}$ 增殖趋势相似, 且在 $\mathrm{DC}$ 与 $\mathrm{PBMC}$ 细胞数量相近时 $(1 ： 1)$, 细胞明 显增殖两次。（图5a、b)。

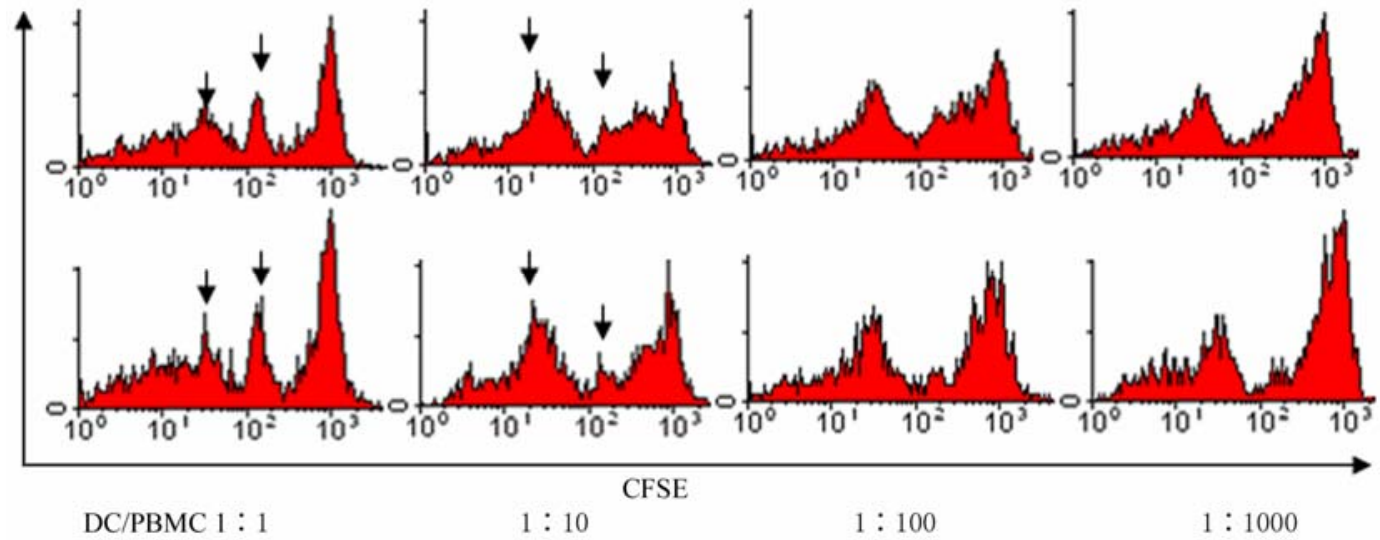

图 5 混合淋巴细胞培养检测成熟 $\mathrm{DC}$ 激活 $\mathrm{T}$ 细胞增殖的能力

Fig. 5 The stimulatory capacity of mature DC in the primary allogeneic MLR. a: 2+2成熟DC, b: 6+2成熟 $\mathrm{DC}$ 。a: fast $\mathrm{mDC}, \mathrm{b}$ : standard $\mathrm{mDC}$

\section{3 讨 论}

体外诱导DC的关键在于：高纯度的前体细胞， 培养过程中保持细胞高存活力, 稳定的培养体系和 恰当的诱导因子，以及合适的培养时间。本实验在 收集CD14 ${ }^{+}$细胞时, 放弃了传统贴壁的办法, 而采 用MACS 分选系统, 通过在磁场中阳性篮选, 快速 而且高纯度的收集单核细胞。避免了PBMC贴壁培 养过程和悬浮细胞淘洗过程, 减少了对细胞的物理 损伤, 保证了 $\mathrm{CD} 14^{+}$单核细胞的纯度与活力。体外 培养过程中, 同时也发现单核细胞培养72 h后, 细 胞开始大量死亡。为提高得率, 本实验通过细胞因 子搭配和培养时间调整, 使单核细胞在 $48 \mathrm{~h}$ 实现分 化, $96 \mathrm{~h}$ 完成成熟, 提高了单核细胞向DC诱导的效 率。

在诱导 DC的细胞因子选择方面, 本实验以 Sallusto \& Lanzavecchia(1994) 和 Zhou \& Tedder(1996)建立的分阶段两步诱导DC的方法为基 础, 用GM-CSF、IL-4、rTNF- $\alpha$ 将CD14 ${ }^{+}$单核细胞 向DC诱导，但在培养过程中出现了大量巨噬细胞， 且成熟阶段的特征表型CD83表达较低、仅占 $30 \%$ 左 右。通过调整细胞因子IL-4和GM-CSF在培养体系 的浓度用量, 证实GM-CSF是诱导DC的主要因子, 而 IL-4 的作用是阻止单核细胞分化为巨噬细胞 (Caux et al，1992），只需要保持比GM-CSF更低
的浓度。成熟活化阶段，则尝试将rTNF- $\alpha$ 改为LPS （KoskiLyakh \& Rice，2001）或者rTNF- $\alpha$ 、IL-1 $\beta$ 、 IL-6、PGE 2 多种因子联合活化iDC成熟 (Feuerstein et al，2000)。结果发现GM-CSF (1000 U/mL)、IL-4 ( $500 \mathrm{U} / \mathrm{mL}$ ) 和 $r T N F-\alpha 、 I L-1 \beta 、 I L-6 、 \mathrm{PGE}_{2}$ 联合 激活能更快速高效的诱导 DC。

在诱导的时间上，通过在不同时间点收集细 胞, 分析比较其形态、表型和功能, 寻找诱导 DC 所需的恰当时间。结果发现在不成熟因子 rGM-CSF、rIL-4 作用下, 分化 $24 \mathrm{~h}$ 后细胞仍高表达 CD14，低表达 CD1a，能够吞噬 dextran，与文献报 道基本相符, 表面标志的表达水平不及典型 iDC 高

（Jarnjak-Jankovic et al，2007）。但 48 小时后细 胞已具有较高吞噬 dextran 的能力, 且 CD14 表达减 少，，HLA-DR、CD40、CD1a、CD80、DC-SIGN 的表达水平升高, 与诱导 6 天的 $\mathrm{iDC}$ 表型基本一致, 仅 DC-SIGN 的表达相对略低。综合细胞表型和抗 原摄取功能, 本实验将诱导 $\mathrm{iDC}$ 的时间改变为 2 天。 成熟阶段，仍以传统法为标准，用 rTNF- $\alpha$ 、IL- $1 \beta$ 、 IL-6、PGE 2 联合激活 2 天确保 iDC 的充分活化和成 熟。形态、表型、功能结果表明活化后的细胞具备 典型 $\mathrm{mDC}$ 的特征, 细胞突起增多, 高表达 HLA-DR、 CD40、CD86、CD83，能激活 T 细胞增殖。

综上所述，“2+2”法通过缩短分化阶段的时间 
和选择成熟阶段的细胞因子组合诱导的 DC, 在未 成熟和成熟阶段，其形态、表型和功能都具备了传 统法刺激形成的 DC 的特征。这种方法缩短了实验

\section{参考文献:}

Campbell-Anson RE, Kentor D, Wang YJ, Bushnell KM, Li Y, Vence LM, Radvanyi LG. 2008. A new approach for the large-scale generation of mature dendritic cells from adherent PBMC using roller bottle technology [J]. J Immune Based Therapies and Vaccines, 6(1): 1-10.

Caux C, Dezutter-Dambuyant C, Schmitt D, Banchereau J. 1992. GM-CSF and TNF-Alpha cooperate in the generation of dendritic langerhans cells [J]. Nature, 360(6401): 258-261.

Daue M, Obermaier B, Herten J, Haerle C, Pohl K, Rothenfusser S, Schnurr M, Endres S, Eigler A. 2003. Mature dendritic cells derived from human monocytes within 48 hours: A novel strategy for dendritic cell differentiation from blood precursors [J]. $J$ Immunol, 170(8): 4069-4076.

Feuerstein B, Berger TG, Maczeka C, Ro“dera C, Schreiner D, Hirsch U, Haendle I, Leisgang W, Glaser A, Kussc O, Diepgenc T, Schulera G, Thurnera BS. 2000. A method for the production of cryopreserved aliquots of antigen-preloaded, mature dendritic cells ready for clinical use [J]. J Immunol Methods, 245(1-2): 15-29.

Jarnjak-Jankovic S, Hammerstad H, Sæbøe-Larssen S, Kvalheim G, Gaudernack G. 2007. A full scale comparative study of methods for generation of functional dendritic cells for use as cancer vaccines [J]. Bmc Cancer, 7(119):2-9.

Jeras M, Bergant M, Repnik U. 2005. In vitro preparation and functional assessment of human monocyte-derived dendritic cells-potential antigen-specific modulators of in vivo immune responses [J]. Transpl Immunol, 14(3-4): 231-244.

Joeri ST, Corthals AJ, t Neyns B, Heirman C, Breckpot K, Thielemans K, Bonehill A. 2007. Current approaches in dendritic cell generation and future implications for cancer immunotherapy [J]. Cancer Immunol Immu, 56(10): 1513-1537.

Koski GK, Lyakh LA, Rice NR. 2001. Rapid lipopolysaccharide-induced differentiation of $\mathrm{CD}_{14}{ }^{+}$monocytes into $\mathrm{CD} 83^{+}$dendritic cells is modulated under serum-free conditions by exogenously added IFN-r
周期，节省实验成本，为肿瘤 $\mathrm{DC}$ 疫苗研制和 $\mathrm{DC}$ 功能研究提供了一种更便捷的途径。

and endogenously produced IL-10 [J]. Eur J Immunol, 31(14): 3773-3781.

Landi A, Babiuk LA, Hurk S. 2007. High transfection efficiency, gene expression, and viability of monocyte-derived human dendritic cells after nonviral gene transfer [J]. J Leukocyte Bio, 82(4): 849-860.

Obermaier B, Dauer M, Herten J, Schad K, Endres S, Eigler A. 2003. Development of a new protocol for 2-day generation of mature dendritic cells from human monocytes[J]. Biol Proced Online, 5(1): 197-203.

Randolph GJ, Beaulieu S, Lebecque S, Steinman RM, Muller WA. 1998. Differentiation of monocytes into dendritic cells in a model of transendothelial trafficking [J]. Science, 282(5388): 480-483.

Romani N, Reider D, Heuer M, Ebner S, Kampgen E, Eibl B, Niederwieser D, Schuler G. 1996. Generation of mature dendritic cells from human blood: An improved method with special regard to clinical applicability [J]. J Immunol Methods, 196(2): 137-151.

Sallusto F, Lanzavecchia A. 1994. Efficient presentation of soluble-antigen by cultured human dendritic cells is maintained by granulocyte-macrophage colony-stimulating factor plus interleukin-4 and down-regulated by Tumor-Necrosis-Factor-Alpha [J]. J Exp Med, 179(4): 1109-1118.

Santini SM, Lapenta C, Logozzi M, Parlato S, Spada M, Pucchio TD, Belardelli F. 2000. Type I interferon as a powerful adjuvant for monocyte-derived dendritic cell development and activity in vitro and in Hu-PBL-SCID mice [J]. J Exp Med, 191(10): 1777-1788.

Sousa CR. 2006. Dendritic cells in a mature age [J]. Nat Rev Immunol, 6(6): 476-483.

Zhou LJ, Tedder TF. 1996. CD14 ${ }^{(+)}$blood monocytes can differentiate into functionally mature $\mathrm{CD} 83^{(+)}$dendritic cells [J]. Proceedings of the National Academy of Sciences of the United States of America, 93(6): 2588-2592. 